Ann. Zootech., I979, 28 (4) 465-47I.

Note

\title{
Altérations du rythme électrique de base de l'antre gastrique après diverses vagotomies chez le Porc
}

\author{
Béatrice DARCY, J.-P. IAPLACE et P.-A. VILI,IERS \\ Laboratoire de Physiologie de la Nutrition, \\ Centre national de Recherches zootechniques, I.N.R.A., \\ 78350 Jouy-en-Josas (France)
}

\begin{abstract}
Résumé
Chez 8 porcs préalablement équipés d'électrodes implantées chroniques, les modifications éventuelles de la fréquence des activités électriques de contrôle au niveau de l'antre gastrique sont étudiées après diverses vagotomies. Les interventions réalisées visent à réduire de façon graduelle le volume des afférences et/ou efférences vagales, issues des ou parvenant aux organes postdiaphragmatiques. Aucune anomalie de rythme n'est enregistrée après déafférentation subtotale, ni après la réalisation conjuguée de 2 vagotomies tronculaires, l'une cervicale et l'autre au niveau du diaphragme. Par contre, de fréquents défauts d'électrogénèse antrale sont observés après déafférentation totale. Ces anomalies semblent résulter de la suppression totale des afférences vagales. Il n'est cependant pas possible d'exclure totalement un rôle de la réduction concomitante de certaines efférences. L'évaluation de l'importance anatomo fonctionnelle des divers contingents de fibres vagales s'avère nécessaire pour l'analyse de ces phénomènes.
\end{abstract}

\section{Introduction}

La disposition anatomique particulière des voies vagales chez le Porc autorise une déafférentation chirurgicale sélective (DARCY et al., I979). Celle-ci permet de supprimer l'intéroception d'origine digestive, empruntant la voie vagale afférente, tout en préservant l'intéroception d'origine cardiopulmonaire et un certain volume d'efférences vagales. Toutefois, en raison de l'existence de rameaux de communication entre le tronc vagal droit et son homologue gauche sur leur trajet intra thoracique, la déafférentation des organes postdiaphragmatiques peut être plus ou moins complète (LAPLACE, I979a). La déafférentation dite subtotale 
entraîne une prolongation de l'hyperactivité électromyographique (E.M.G.) postprandiale de l'antre gastrique (LAPLACE et DARCY, I978; DARCY et al., I979), un renforcement de 1'amplitude des activités rapides antrales particulièrement net lors d'ingestion d'un aliment semi-synthétique (LAPIACE, I979b), et un ralentissement de l'évacuation gastrique (LAPLACE, CUBER et VILLIERS, I979.). La déafférentation totale conduit également à une prolongation de l'hyperactivité E.M.G. postprandiale de l'antre et à une lenteur d'évacuation gastrique pouvant évoluer vers une véritable stase, 1'anorexie et la mort dans $30 \mathrm{p}$. IOO des cas (I,APIACE, r979a).

Comme on peut le constater à l'aide des schémas de la figure I, la déafférentation subtotale préserve un volume d'efférences équivalant à celui qui reste après une vagotomie tronculaire unilatérale au niveau du diaphragme. Par contre, la déafférentation totale est susceptible de diminuer encore 1'importance du contingent de fibres efférentes parvenant aux organes postdiaphragmatiques. Aussi est-il nécessaire d'évaluer le rôle éventuel de la réduction des efférences dans la particulière gravité des conséquences de la déafférentation totale.

\section{Matériel et méthodes}

Huit porcs de race Large White, pesant environ $60 \mathrm{~kg}$, sont utilisés. Sous anesthésie générale à l'halothane, des électrodes sont implantées dans les couches musculaires de l'antre terminal $(8 \mathrm{~cm}$ au plus en amont du pylore). Ces animaux en préparation chronique reçoivent 2 repas par jour d'aliment standard à base de céréales. Après l'obtention d'électromyogrammes témoins, ils subissent l'une des interventions ci-après, schématisées dans la figure I :
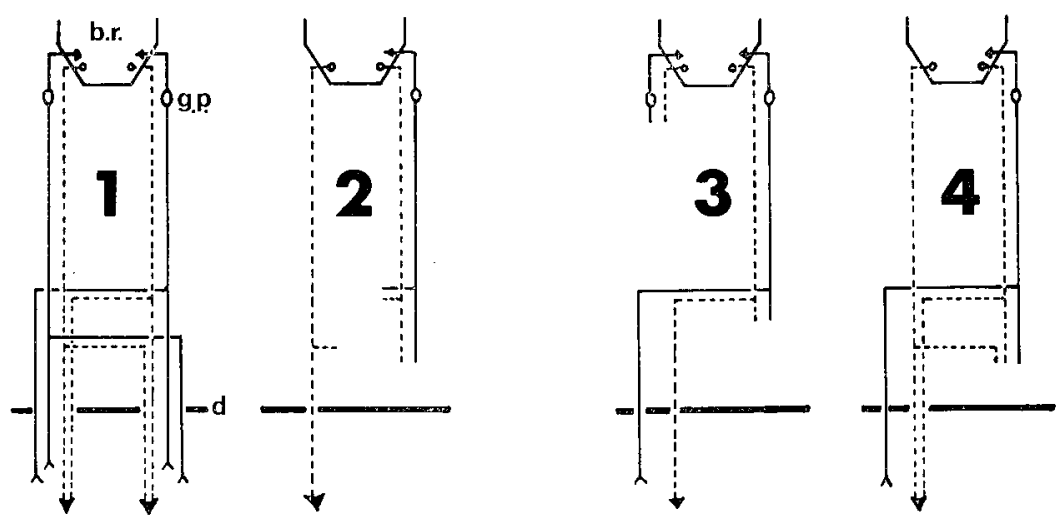

F.IF. I. - Représentation schématique des voies vagales motrices (pointillés) et sensitives (trait continu) chez le porc intact ( $(1)$, après déafférentation vagale totale (2), après association d'une vagotomie tronculaive cervicale gauche et d'une vagotomie tronculaive diaphragmatique droite (3), et apvès déafféventation vagale subtotale (4). b.r. = bulbe rachidien; $g . p .=$ ganglion plexiforme; $d=$ diaphragme.

Diagram of the vagal motor (dotted line) and sensory (straight line) pathways in the intact pig (I), after total vagal deafferentation (2), after combined truncular cervical and truncular diaphragmatic vagotomy (3), and after subtotal deafferentation (4). $b \quad r .=$ medulla oblongata; g. $p .=$ nodose ganglion; $d=$ diaphragm. 
- déafférentation vagale subtotale par ablation du ganglion plexiforme gauche et vagotomie tronculaire droite au niveau du diaphragme (schéma 4);

-- vagotomie tronculaire cervicale gauche associée à une vagotomie tronculaire droite au niveau du diaphragme (schéma 3);

- déafférentation vagale totale, la section des branches d'interconnexion des vagues thoraciques s'ajoutant aux 2 temps de la déafférentation subtotale (schéma 2);

- vagotomie tronculaire bilatérale au niveau du diaphragme; celle-ci n'est pas schématisée, étant donné qu'elle supprime la totalité des voies vagales afférentes et efférentes distalement au diaphragme.

La représentation schématique des voies vagales dans la figure I ne préjuge évidemment pas de l'importance anatomique respective de chacun des contingents qui n'est pas encore à ce jour déterminée chez le Porc. A noter également que les interventions contralatérales correspondant aux schémas 2,3 et 4 n'ont pas été mises en œuvre jusqu'ici.

\section{Résultats et discussion}

Ia déafférentation vagale subtotale (fig. I-4), qui préserve le maximum d'efférences motrices destinées aux organes post diaphragmatiques n'est jamais suivie d'anomalies du rythme électrique de base antral. L'association d'une vagotomie tronculaire cervicale gauche et d'une vagotomie tronculaire droite au niveau du diaphragme (fig. I-3), laissant subsister le même contingent afférent que la déafférentation subtotale, mais réduisant plus le volume des efférences, n'entraîne pas non plus d'anomalies de la fréquence des activités électriques de contrôle. Ia déafférentation vagale totale (fig. I-2) qui préserve le même volume réduit d'efférences que dans le cas précédent en supprimant toutes les voies sensitives, est par contre suivie de l'apparition de fréquentes aberrations du rythme électrique de base antral. Ces anomalies (fig. 2) consistent en l'absence de une ou plusieurs activités électriques de contrôle consécutives et surviennent aussi bien chez le porc à jeûn qu'en période post prandiale. Ce défaut d'électrogenèse dure le plus souvent environ I $\mathrm{mn}$, mais a été constaté pendant $6 \mathrm{mn}$ consécutives au $8 \mathrm{e}$ jour post opératoire chez un porc.

Par rapport aux deux autres interventions, il semble donc que la perte de l'ultime contingent sensitif vagal (déafférentation totale) soit directement responsable des anomalies de la fréquence des activités électriques de contrôle antrales. On notera que, par ailleurs, les trois types d'opération ont des effets analogues quant à la durée de l'hyperactivité E.M.G. postprandiale de l'antre (LAPLACE, I979a). Pourtant, 1'importance anatomique et la destination des divers contingents de fibres vagales n'étant pas à ce jour précisées, et les interventions contralatérales n'ayant pas été réalisées, une autre hypothèse doit être considérée. En effet, la déafférentation totale (fig. I-2) conduit, par rapport à la déafférentation subtotale (fig. I-4), à la suppression du contingent efférent qui atteint le vague abdominal gauche à partir du vague thoracique droit. Or c'est précisément ce contingent efférent qui se trouve préservé dans le cas de l'opération schématisée à la figure I-3, intervention qui n'est pas suivie de l'apparition d'anomalies de la fréquence des activités de contrôle. On ne peut donc exclure l'éventualité que ce 
contingent efférent soit distribué précisément à l'antre gastrique et que sa suppression soit responsable des anomalies observées.

Quelle que soit l'explication véritable des aberrations du rythme électrique de base (suppression totale de toute afférence ou perte d'un contingent efférent à destination spécifiquement antrale), leur existence implique nécessairement (LAPI,ACE et RoMAx, I979) des désordres de la propagation des contractions antrales qui ne peuvent qu'aggraver l'inefficacité relative de la motricité antrale déjà présente après déafférentation subtotale (LAPLACE, CUBER et VILLIERS, I979). Ce cumul d'effets pourrait rendre compte du fait que la rétention gastrique, en l'absence de toute précaution diététique, est mortelle dans 30 p. Ioo des cas après déafférentation totale. Le pronostic serait certainement amélioré par la mise en œuvre, classique, d'une alimentation liquide, mais l'usage de l'aliment standard relativement grossier sert ici de révélateur des troubles fonctionnels.

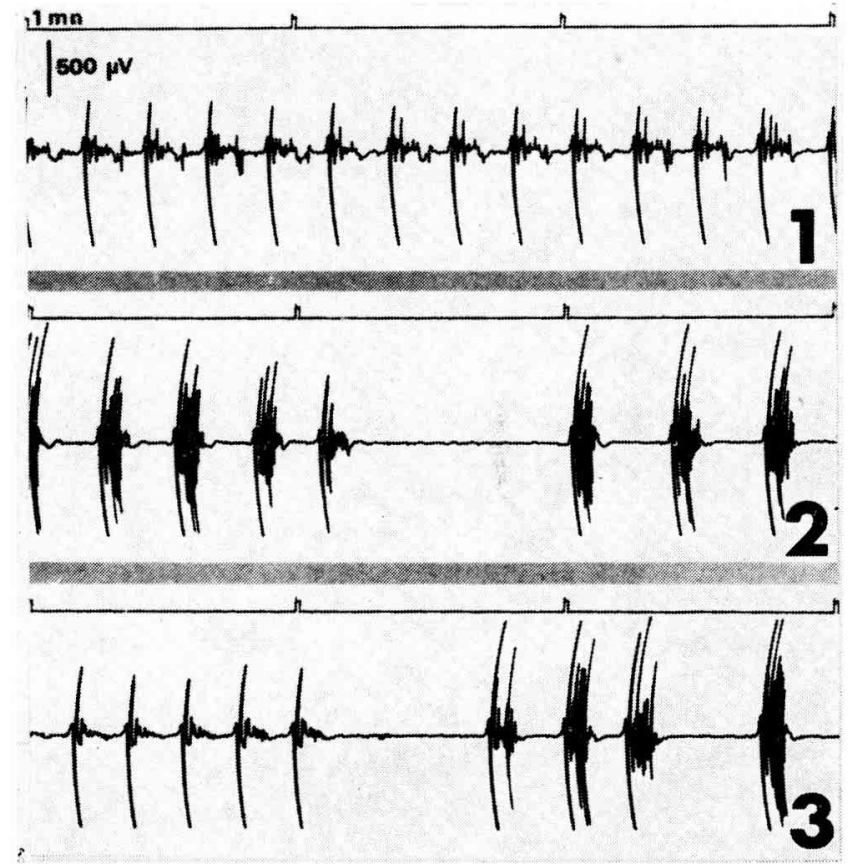

Fic. 2. - Electromyogrammes chroniques de l'antre gastrique à $3 \mathrm{~cm}$ du pylore chez un porc avant (I) et après déalférentation vagale totale (2 et 3). Enregistrement on dérivation bipolaire; constante de temps -- 0,03 sec.

Electromyographic bipolar recording from the gastric antrum (3 $\mathrm{cm}$ above pylorus) in a pig before (I) and after total deafferentation (2 et 3) time constant $-0.03 \mathrm{sec}$.

A titre comparatif, on notera que la vagotomie tronculaire bilatérale au niveau du diaphragme, supprimant toute intéroception et tout contrôle par le vague des organes postdiaphragmatiques, conduit à une très importante rétention gastrique éventuellement fatale et à l'apparition de très fréquentes aberrations du rythme électrique de base antral. Mais celles-ci consistent, quel que soit l'état de réplétion digestive, soit en l'absence de une ou plusieurs activités de contrôle comme après déafférentation totale, soit plus souvent (fig. 3) en une succession rapide d'ondes à une fréquence habituellement double de la fréquence normale du rythme électrique 
de base. L'existence, au moins dans les premiers jours consécutifs à une vagotomie bilatérale, d'une importante perturbation du rythme électrique de base (modifications d'amplitude, de fréquence et de vitesse de propagation) a déjà été signalée (NELSEN et al., Ig67; KELIY et Code, I969; PaPAsova et ATANAssova, I972; MIOLAN, I974). L'observation récente chez le chien (MIOLAN et RoMAN, I978) de l'interruption prolongée des décharges vagales efférentes parallèlement à 1'apparition d'ondes atypiques de fréquence élevée suggère qu'il s'agit là d'un phénomène caractérisant la privation d'efférences. Dans le cas de nos porcs soumis à vagotomie tronculaire bilatérale au niveau du diaphragme, il reste difficile d'affirmer que cette anomalie est véritablement caractéristique de la privation complète d'efférences vagales, non seulement en raison du faible nombre d'animaux concernés, mais aussi du fait que la suppression totale concomitante de toute afférence conduirait sans doute aussi à une forte réduction du volume des efférences.

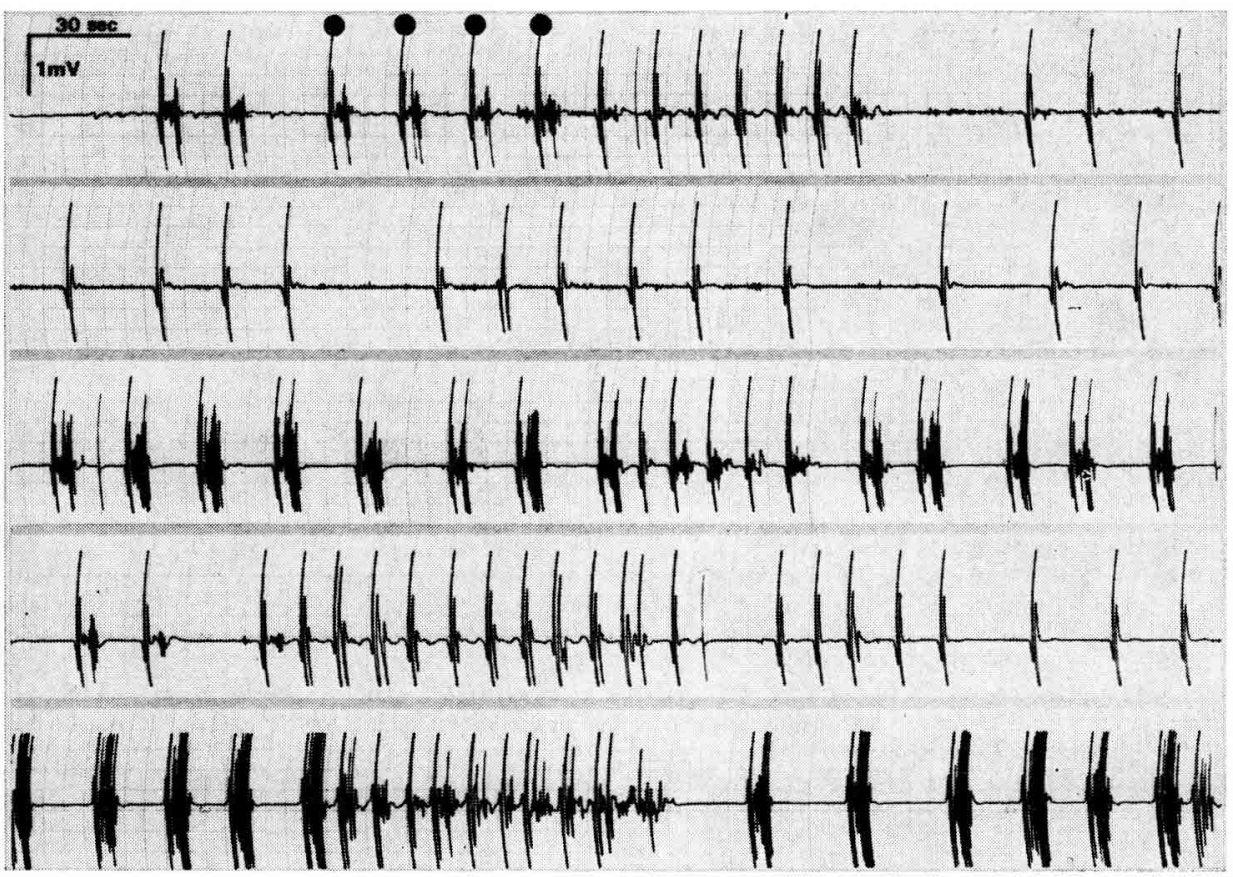

FIG. 3. - Electromyogrammes chroniques de l'antre gastrique à $5 \mathrm{~cm}$ du pylore chez un porc après vagotomie tronculaire bilatérale à hauteur du diaphragme. Les tracés sont vecueillis, de haut en bas, avant le repas du matin, juste après le début du vepas, apvès achèvement de celui-ci, et enfin 3 et $5 \mathrm{~h}$ plus tard. Envegistrements en dérivation bipolaive; constante de temps $=0, o_{3}$ sec. Les points indiquent la fréquence normale du rythme électrique de base.

Electromyographic bipolar recording from the gastric antrum (5 $\mathrm{cm}$ above pylorus) in a pig after bilateral truncular vagotomy at the diaphragm. The panels from top to bottom were recorded: before the morning meal, just after the beginning of the meal, after it was finished, and later on ( 3 and $5 \mathrm{~h}$ after its end): time constant $=0.03 \mathrm{sec}$. The dots indicate the normal jrequency of the basic electrical rhythm.

Quoiqu'il en soit, ce type particulier d'aberration (ondes de fréquence élevée) n'ayant pas été à ce jour enregistré après déafférentation totale, on pourrait admettre que le contingent moteur préservé dans cette dernière intervention est 
suffisant pour éviter ce que DANIEL et IRWIN (I968) ont qualifié de "pattern " de dominance sympathique.

Enfin, il semble que d'importantes variations individuelles existent dans ce domaine. Certains pores présentent d'emblée, de façon quasi permanente et persistante, les anomalies décrites ci-dessus à la suite de la vagotomie tronculaire bilatérale. Mais d'autres en sont totalement exempts, ce qui reste difficilement explicable. Une variabilité similaire existe à l'égard des anomalies du rythme électrique de base antral après déafférentation totale, phénomène qui pourrait être en relation avec une importance anatomo-fonctionnelle variable des divers contingents de fibres.

En conclusion: L'existence effective d'altérations du rythme électrique de base antral après déafférentation vagale totale pourrait rendre compte de la particulière gravité des conséquences de cette intervention par rapport à celles de la déafférentation vagale subtctale. Mais il reste difficile d'affirmer que ces altérations du rythme électrique de base résultent exclusivement de la suppression totale des afférences vagales. De fait, l'évaluation de l'importance anatomo fonctionnelle des divers contingents de fibres et l'identification de leur distribution viscérale seront nécessaires pour dissocier les effets respectifs de la privation d'afférences et de la réduction du volume des efférences, celles-ci étant affectées par celles là.

Accepté pour publication en novembre 1979.

\section{Summary}

Alterations of the basic electrical rhythm of the gastric antrum after various vagotomies in the pig.

In 8 pigs with chronic electrodes previously implanted on the gastric antrum, the possible change of the frequency of the electrical control activities was studied after various operations more or less reducing the vagal afferences from and/or efferences to the post diaphragmatic viscera (fig. I). No thythm disturbance was seen after subtotal deafferentation, nor after combined cervical truncular plus diaphragmatic truncular vagotomies. But frequent lack of electrical control activities was observed in the antrum after total deafferentation. The total suppression of vagal afferences appeared to be responsible for that. Nevertheless a possible role of the associated reduction of the vagal efferences cannot be excluded. The anatomical and functional evaluation of the various vagal bundles is required for further advance.

\section{Références bibliographiques}

DANIEL E. E., IRWIN J., I968. Electrical activity of gastric musculature, I969-I984, chap. 96. In Handbook of physiology, Sect. 6 Alimentary canal, Vol. 4 Motility, Amer. Physiol. Soc. Ed., Washington.

DARCY B., FAI EMPIN M., I,APIACE J.-P., RoussfaU J.-P., I979. Impottance de la voie vagale sensitive : Recherche d'une technique de déafférentation sélective chez le porc et le mouton. Ann. Biol. anim. Bioch. Biophys., 19, 881-888.

KELLY K. A., CODE C. F., I 969 . Effect of transthoracic vagotomy on canine gastric electrical activity. Gastroenterology, 57, $5 \mathrm{I}-58$.

LAPLACE J.-P., I979a. Surgical deprivation of vagal afferences from gastro intestinal tract of the pig. Clinical and EMG studies. $7^{\text {th }}$ Int. Symposium on Gastro intestinal Motility, Iowa City (in Press). 
LAPLACE J.-P., I979b. Motricité et évacuation gastriques chez le Porc. Med et $N u t r ., 15,17-23$. LAPLACE J.-P., CUBER J.-C., Villiers P. A., I979. Fivacuation gastrique après déafférentation vagale subtotale chez le Porc. Ann. Zootech., 28, 459-463.

LAPLACE J.-P., DARCY B., I978. Gastric motility in the Pig after surgical interruption of vagal afferent pathways. K. A. 44 , in 5 th Int. Pig Vet. Soc. Congress, Zagreb.

LAPLACE J.-P., ROMAN C., 1979. Activités de la musculature gastro intestinale et mouvements des contenus digestifs. Ann. Biol. anim. Bioch. Biophys., 19, 849-879.

MIOLAN J.-P., I974. La motricité de l'estomac et du sphincter cesophagien inférieur, étude électromyographique; rôle de l'innervation extrinsèque. Thèse Doct. Spécial. Physiol. Anim., Univ. Aix-Marseille III.

Miolan J.-P., ROMAN C., 1978. Discharge of efferent vagal fibers supplying gastric antrum: indirect study by nerve suture technique. Am. J. Physiol., 235, E $366-\mathrm{E} 373$.

Nel.sen T. S., EigenbrodT E. H., Keoshian L. A., Bunker C., Johnson L., I967. Alterations in muscular and electrical activity of the stomach following vagotomy Arch. Surg., 94, 82I835 .

Papasova M., Atanassova E., 1972. Changes in the bioelectric activity of the stomach after bilateral transthoracal vagotomy. Bulgarian Acad. Sci. 14, I2I-I33. 